\title{
Erratum to: Integration by Parts for Perron Type Integrals of Order 1 and 2 in Riesz Spaces
}

\author{
A. Boccuto, A. R. Sambucini, and V. A. Skvortsov
}

\begin{abstract}
We note that the proof of differentiability of the integral function $I_{2}$ given in [1, Theorem 7.9] needs some corrections which we present here.
\end{abstract}

Mathematics Subject Classification (2000). 28B15, 28B05, 28B10.

Keywords. Perron integral, major and minor functions.

\section{Erratum to: Results. Math. (2007) 51:5-27 DOI 10.1007/s00025-007-0254-4}

The proof of differentiability of the integral function $I_{2}$ in [1, Theorem 7.9] is based on the sufficient condition for global differentiability given in [2, Theorem 4.3] and stated in the paper as Proposition 4.5. But this last statement is not justified in [2, Theorem 4.3] properly. In fact the following question seems to be open: if the "componentwise differentiability" in the complement of a meager set does imply global differentiability, where the involved "components" are taken according to the Maeda-Ogasawara-Vulikh representation theorem for Riesz spaces. To overcome this gap we present here a new proof of differentiability of the integral function $I_{2}$, which is based on a comparison theorem for $(g)$-differentiable functions (Lemma 1 below), and on a Cauchy-type criterion ([2, Theorem 3.10]), which states that the global limit $(g) \lim _{h \rightarrow 0} \phi(x, h)$ (in $[a, b]$ ) exists in $R$ if and only if there exists an

Supported by University of Perugia and RFFI-05.01.00206.

The online version of the original article can be found under doi:10.1007/s00025-007-0254-4. 
(o)-net $\left(p_{\gamma}\right)_{\gamma \in \Gamma}$ (that is a net with $p_{\gamma} \downarrow 0$ ) with the property that, for all $\gamma \in \Gamma=\left(\mathbb{R}^{+}\right)^{[a, b]}$, we have:

$$
\begin{aligned}
& \sup _{x \in[a, b]}(\sup \{\phi(x, h): \quad 0<|h| \leq \gamma(x)\}-\inf \{\phi(x, l): \quad 0<|l| \leq \gamma(x)\}) \\
& \quad=\sup _{x \in[a, b]}(\sup \{|\phi(x, h)-\phi(x, l)|: \quad 0<|h|,|l| \leq \gamma(x)\}) \leq p_{\gamma} .
\end{aligned}
$$

The technical comparison lemma is as follows:

Lemma 1. Let $f_{j}:[a, b] \rightarrow R, j=1,2,3$, be such that $f_{1}, f_{2}$ are $(g)$-differentiable in $[a, b]$ and $f_{1}-f_{3}, f_{3}-f_{2}$ are convex in $[a, b]$, then $f_{3}$ is $(g)$-differentiable in $[a, b]$.

Proof. Fix arbitrarily $x, h, l$ with $h, l \in \mathbb{R} \backslash\{0\}, x, x+h, x+l \in[a, b]$. By convexity of $f_{1}-f_{3}$, when $h \geq l$ we get

$$
\frac{\left(f_{1}-f_{3}\right)(x+h)-\left(f_{1}-f_{3}\right)(x)}{h} \geq \frac{\left(f_{1}-f_{3}\right)(x+l)-\left(f_{1}-f_{3}\right)(x)}{l},
$$

and hence

$$
\frac{f_{1}(x+h)-f_{1}(x)}{h}-\frac{f_{1}(x+l)-f_{1}(x)}{l} \geq \frac{f_{3}(x+h)-f_{3}(x)}{h}-\frac{f_{3}(x+l)-f_{3}(x)}{l} .
$$

Analogously, using convexity of $f_{3}-f_{2}$, we obtain, for $h \geq l$ :

$$
\frac{f_{3}(x+h)-f_{3}(x)}{h}-\frac{f_{3}(x+l)-f_{3}(x)}{l} \geq \frac{f_{2}(x+h)-f_{2}(x)}{h}-\frac{f_{2}(x+l)-f_{2}(x)}{l} \text {. }
$$

If $h<l$, by reversing the above inequalities we have:

$$
\begin{aligned}
& \frac{f_{1}(x+h)-f_{1}(x)}{h}-\frac{f_{1}(x+l)-f_{1}(x)}{l} \\
& \leq \frac{f_{3}(x+h)-f_{3}(x)}{h}-\frac{f_{3}(x+l)-f_{3}(x)}{l} \\
& \leq \frac{f_{2}(x+h)-f_{2}(x)}{h}-\frac{f_{2}(x+l)-f_{2}(x)}{l} .
\end{aligned}
$$

In any case we get:

$$
\begin{aligned}
0 & \leq\left|\frac{f_{3}(x+h)-f_{3}(x)}{h}-\frac{f_{3}(x+l)-f_{3}(x)}{l}\right| \\
& \leq \sum_{j=1}^{2}\left|\frac{f_{j}(x+h)-f_{j}(x)}{h}-\frac{f_{j}(x+l)-f_{j}(x)}{l}\right| .
\end{aligned}
$$

Since $f_{1}, f_{2}$ are $(g)$-differentiable in $[a, b]$, by (1) applied to $\phi(x, h)=$ $\frac{f_{j}(x+h)-f_{j}(x)}{h}, j=1,2$, two $(o)$-nets $\left(p^{(j)}\right)_{\gamma \in \Gamma}$ can be found, with

$$
\sup _{x \in[a, b]}\left(\sup _{0<|h|,|l| \leq \gamma(x)}\left|\frac{f_{j}(x+h)-f_{j}(x)}{h}-\frac{f_{j}(x+l)-f_{j}(x)}{l}\right|\right) \leq p_{\gamma}^{(j)}
$$


for all $\gamma \in \Gamma$. From this and from (2) we obtain

$$
0 \leq\left|\frac{f_{3}(x+h)-f_{3}(x)}{h}-\frac{f_{3}(x+l)-f_{3}(x)}{l}\right| \leq p_{\gamma}^{(1)}+p_{\gamma}^{(2)}
$$

for all $\gamma \in \Gamma$, whenever $x, x+h, x+l \in[a, b]$ and $0<|h|,|l| \leq \gamma(x)$. Since the net $\left(p_{\gamma}^{(1)}+p_{\gamma}^{(2)}\right)_{\gamma \in \Gamma}$ is an (o)-net, then, again by (1) used for $\phi(x, h)=$ $\frac{f_{3}(x+h)-f_{3}(x)}{h}$, we get $(g)$-differentiability of $f_{3}$ in $[a, b]$.

Now we are ready to give the new proof of differentiability of the integral function $I_{2}$. In fact we replace the text on page 18 of [1, Theorem 7.9] from line 5 up to the end of the page 18 with the following one. The new fragment of the proof of $[1$, Theorem 7.9$]$ is:

Proof. Now we prove the differentiability of $I_{2}$. Let $\Psi$ (resp. $\Phi$ ) be any fixed major (minor) function of order 2 for $f$. We know that $\Psi, \Phi$ are $(g)$-differentiable in $[a, b]$ and $\Psi-I_{2}, I_{2}-\Phi$ are convex in $[a, b]$. In fact, for example, if we take $J:=I_{2}-\Phi$, observe that $J(b)=\inf _{\Psi}[\Psi(b)-\Phi(b)]$, where the infimum is taken along all the major functions $\Psi$. Fix any pair $(x, y)$ in $[a, b]$, choose any number $c \in] 0,1\left[\right.$, and any major function $\Psi$. Writing $\Psi_{1}:=\Psi-\Phi$, thanks to convexity of $\Psi_{1}$ and since $J-\Psi_{1}=I_{2}-\Psi$ is decreasing, we have:

$$
\begin{aligned}
c J(x)+(1-c) J(y)= & c \Psi_{1}(x)+(1-c) \Psi_{1}(y)+c\left[J(x)-\Psi_{1}(x)\right] \\
& +(1-c)\left[J(y)-\Psi_{1}(y)\right] \\
\geq & \Psi_{1}(c x+(1-c) y)+c\left[J(b)-\Psi_{1}(b)\right] \\
& +(1-c)\left[J(b)-\Psi_{1}(b)\right] \\
\geq & J(c x+(1-c) y)+\left[J(b)-\Psi_{1}(b)\right] .
\end{aligned}
$$

Now, taking the supremum with respect to $\Psi$, we get

$$
c J(x)+(1-c) J(y) \geq J(c x+(1-c) y) .
$$

So, $I_{2}-\Phi$ is convex. Thus, $(g)$-differentiability of $I_{2}$ is an immediate consequence of Lemma 1 with $f_{1}=\Psi, f_{2}=\Phi, f_{3}=I_{2}$.

\section{References}

[1] Boccuto, A., Sambucini, A.R., Skvortsov, V.A.: Integration by parts for Perron type integrals of order 1 and 2 in Riesz spaces. Results. Math. 51, 5-27 (2007)

[2] Boccuto, A., Skvortsov, V.A.: Remark on the Maeda-Ogasawara-Vulikh representation theorem for Riesz spaces and applications to Differential Calculus. Acta Math. (Nitra) 9, 13-24 (2006) 
A. Boccuto and A. R. Sambucini

Dipartimento di Matematica e Informatica

Università degli Studi di Perugia

Via Vanvitelli, 1

06123 Perugia

Italy

e-mail: boccuto@dipmat.unipg.it;

boccuto@yahoo.it;

matears1@unipg.it

V. A. Skvortsov

Department of Mathematics

Moscow State University

119992 Moscow

Russia

and

Mathematical Institute

Universytet Kazimierza Wielkiego

85-065 Bydgoszcz

Poland

e-mail: vaskvor2000@yahoo.com 